\title{
History of Stress-related Health Changes: A Cue to Pursue a Diagnosis of Latent Primary Adrenal Insufficiency
}

\author{
Toshihide Yamamoto
}

\begin{abstract}
Objective Routine delays in the diagnosis of primary adrenal insufficiency (PAI) are well known and conceivably attributable to the absence of cues, other than anti-adrenal autoantibodies, to pursue subclinical PAI. Subclinical PAI is latent unless the afflicted patient encounters stress such as an acute illness, surgery, psychosocial burden, etc. It remains to be demonstrated whether a history of stress-related health changes is a useful cue to pursue a diagnosis of latent PAI.

Methods The patients were selected for a history of recurrent symptoms, i.e., gastrointestinal symptoms, fatigue, or lassitude, aggravated by stress and alleviated by the removal of stress, and signs, i.e., weight loss, hypotension, and hyperpigmentation. As the early morning cortisol levels were low or low-normal and the adrenocorticotropic hormone (ACTH) levels were within the reference ranges, provocation tests, i.e., insulininduced hypoglycemia tests and low-dose $(1 \mu \mathrm{g})$ corticotropin tests (LDTs), were used to estimate the hypothalamus-pituitary-adrenal (HPA) axis status. Patients with the HPA axis dysfunction on two provocation tests were supplemented with physiologic doses of glucocorticoids (GCs). The effects of GC supplementation on stress-related health changes were observed.

Results The ACTH levels after insulin-induced hypoglycemia were higher and the cortisol levels were lower in the patients than in the control subjects. The cortisol levels in the patients were increased less significantly by LDT than those observed in the control subjects. Stress-related health changes ceased to recur and signs, i.e., a low body weight, hypotension, and hyperpigmentation, were ameliorated following GC supplementation.
\end{abstract}

Conclusion A history of stress-related health changes is useful as a cue to pursue latent PAI in patients with low or low-normal early morning cortisol levels.

Key words: ACTH, cortisol, insulin-induced hypoglycemia test, insulin tolerance test, low-dose corticotropin test, stress

(Intern Med 53: 183-188, 2014)

(DOI: 10.2169/internalmedicine.53.1156)

\section{Introduction}

Delays in the diagnosis of primary adrenal insufficiency (PAI) are well known $(1,2)$. If subclinical or latent PAI could be detected earlier, then more doctors would pay attention to patients with latent PAI, and afflicted patients would be better managed. Progression to PAI has been documented in patients with positive anti-adrenal autoantibodies (3-6). Tests for anti-adrenal autoantibodies are used to screen for subclinical PAI, especially in Europe. These tests, however, are not available in Japan: therefore other means are needed to pursue a diagnosis of subclinical or latent PAI. The author reported the latent nature of isolated adrenocorticotropic hormone (ACTH) deficiency (IAD) in the sense that most patients with IAD, although doing well in their non-stressful daily lives before being diagnosed and treated, are often brought to the hospitals after developing anorexia, abdominal pain, diarrhea, dehydration, hyponatremia, or hypoglycemia following acute illnesses, overwork, surgery, etc. (henceforth collectively designated as stress) (7). In an endocrinology textbook published in the 
response at 60 minutes was judged based on a cutoff level (CL) of a positive response set at $20 \mu \mathrm{g} / \mathrm{dL}$ (9). For the LDT, blood for cortisol measurement was drawn before, and at 30 and 60 minutes after a 1- $\mu \mathrm{g}$ intravenous injection of corticotropin (Cortrosyn ${ }^{\circledR}$, Daiichi-Sankyo Pharmaceutical Co., Tokyo, Japan). In patients already being supplemented with GC, the GC was withheld for two days prior to the second test, either the short IHT or the LDT. The responses to the LDT were judged based on a 30-minute cortisol level, with a CL of a positive response set at $18 \mu \mathrm{g} / \mathrm{dL}(10)$.

\section{GC supplementation}

The patients were treated with hydrocortisone at a dose of 7.5 to $25 \mathrm{mg}$ per day (50\% for morning use with the remainder divided for noon and evening use). The subjects were instructed to increase the dose of GC whenever expecting stress, i.e., the common cold, uncomplicated gastroenteritis, a hard and long work schedule, a prolonged very hot atmospheric temperature, travel to unfamiliar places, etc.

\section{Results}

Six of the sixteen patients were excluded due to a discordance between the two tests (cf. discussion section). The demographic, clinical and endocrine findings are summarized in Table. Patient 1 developed anorexia and began feeling unwell after several days of hot temperature in the summer. When Patient 2 came to the clinic during an episode of the common cold, the patient's consciousness was obtunded, with an attention span reduced to less than one minute and a blood glucose level of $60 \mathrm{mg} / \mathrm{dL}$. The patient's consciousness became clear following the administration of an i.v. glucose infusion. Patient 3 complained of fatigue and arthralgia and stiffness of the finger joints without deformities. Blood tests for rheumatoid arthritis and systemic erythematodes were negative. The arthralgia was alleviated by a nonsteroidal anti-inflammatory agent. When the patient had a dry cough that lasted approximately one week, she developed orthostatic dizziness and reported cold sweats after working long hours without food intake. Her glucose level three hours after breakfast happened to be $61 \mathrm{mg} / \mathrm{dL}$ on one occasion. Patients 4 and 9 required i.v. fluid supplementation for anorexia and diarrhea on more than two occasions during episodes of the common cold. Patients 5-8 and 10 were troubled by anorexia, abdominal pain, diarrhea, and lassitude when their tight work schedules lasted for days. Recurrent diarrhea in Patients 4 and 5 was attributed to the sequela of past pelvic surgeries. In response to specific inquiries regarding symptoms in reference to concurrent stress, the patients reported that anorexia, abdominal pain, diarrhea, tiredness, and malaise often recurred following stress, the degree of worsening of the symptoms seemed disproportionate to the severity of the stress, and that the recovery was quick after the stress was relieved. Hyperpigmentation, a low body mass index $(\leq 20)$ and low blood pressure (systolic blood pressure $\leq 100 \mathrm{mmHg}$ ) were observed in a few pa- tients.

\section{Endocrine studies}

The early morning cortisol levels were low in two patients $(3.4,4.5 \mu \mathrm{g} / \mathrm{dL})$ and low-normal (5.3-10.1 $\mu \mathrm{g} / \mathrm{dL})$ in the other patients. The early morning ACTH levels of the patients were within the reference range (Table). The changes in the glucose, ACTH, and cortisol levels following insulin injection are illustrated in Fig. 1. The glucose levels were reduced at 30 minutes to below $40 \mathrm{mg} / \mathrm{dL}$ in 13 of the 15 control subjects and in nine patients (Fig. 1, section A). The blood glucose levels at 30 minutes following insulin injection were not statistically different between the patients and the control subjects (Mann-Whitney $U$ test, $\mathrm{p}>0.05$ ). The maximal ACTH levels of 12 of the control subjects were greater than $127 \mathrm{pg} / \mathrm{mL}$ (twice the upper reference range) while the maximal ACTH levels of the other three controls were 79 to $112 \mathrm{pg} / \mathrm{mL}$ (Fig. 1, section B). The maximal ACTH levels at 30 minutes in two of the patients and at 60 minutes in the other patients were in the range of 201 and $415 \mathrm{pg} / \mathrm{mL}$, respectively. The 60-minute ACTH levels of the patients were higher as a group than those of the controls (Mann-Whitney U test, $\mathrm{p}<0.01$ ). The basal and the maximum ACTH levels of two patients with hyperpigmentation were not different from those of the other patients. The 60-minute cortisol levels were increased above the CL (20 $\mu \mathrm{g} / \mathrm{dL}$ ) in all the control subjects, including the subjects whose maximum ACTH level did not rise above twice the upper reference range, while the 60-minute cortisol levels of the patients were not increased above the CL (Fig. 1, section C).

The 30-minute cortisol levels were increased following LDT above the CL set at $18 \mu \mathrm{g} / \mathrm{dL}$ in all control subjects: however, the patients' levels were not raised above the CL (Fig. 2).

\section{Response to GC supplementation}

Gastrointestinal symptoms as well as pain and stiffness of the finger joints ceased to recur within a few weeks after the initiation of GC supplementation. Systolic blood pressure rose above $100 \mathrm{mmHg}$, and two to three months were required for weight gain. Pigmentation of the gum and palm creases became lighter following GC supplementation for more than three months. Patient compliance with GC supplementation was good.

\section{Discussion}

The present patients lacked the usual triad of hyperpigmentation and, low cortisol and elevated ACTH levels and were asymptomatic in their daily lives. Their symptoms appeared to be related to stress: gastrointestinal symptoms occurred following stress and remitted quickly after the stress was relieved, while stress causing anorexia, abdominal pain, diarrhea, or lassitude was not very severe, e.g., a gardenvariety common cold. When asked about symptoms in the 

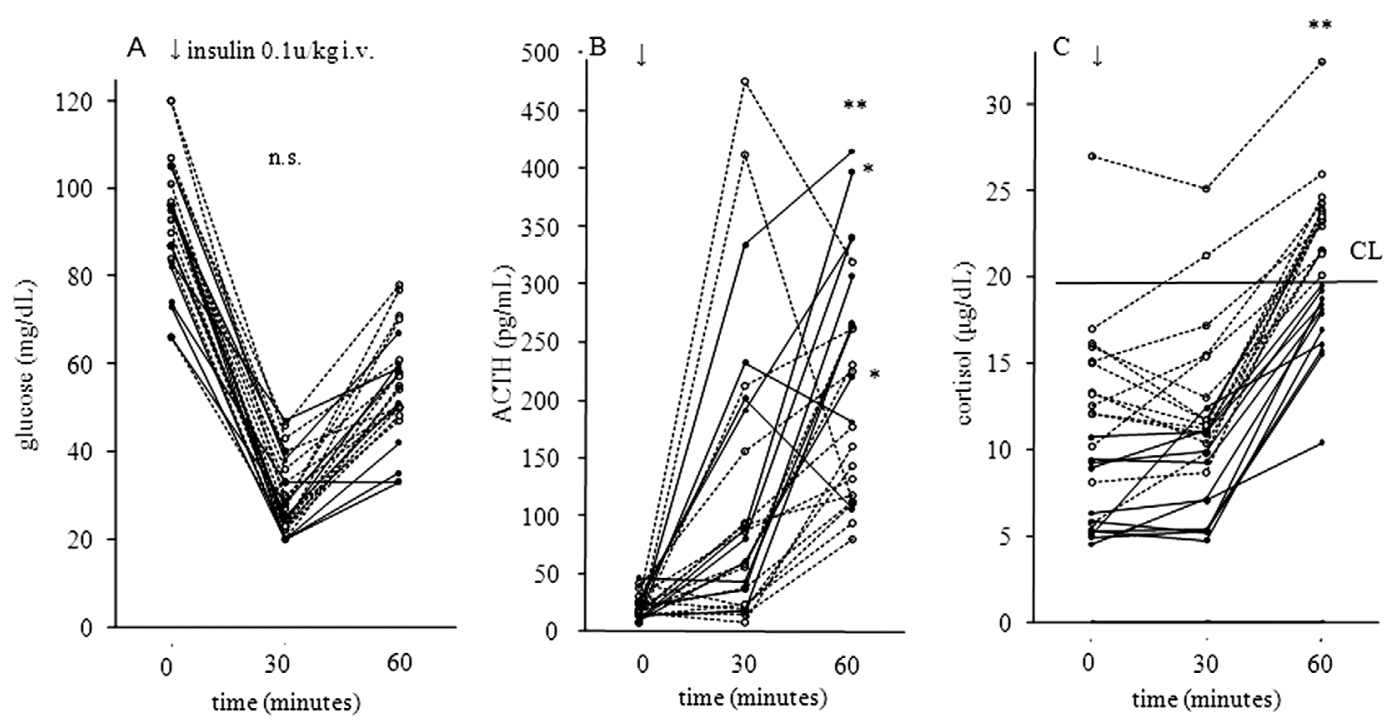

Figure 1. Response of ACTH and cortisol to insulin-induced hypoglycemia in controls and patients with latent primary adrenal insufficiency. Regular insulin (Humalin ${ }^{\circledR}$ ) $0.1 \mathrm{U} / \mathrm{kg}$ was injected after fasting blood sample had been drawn. Data of controls are shown with open circles connected by dotted lines and those of patient with solid circles connected by straight lines. Cutoff line (CL) of positive cortisol response is shown with a horizontal line (section C). ACTH data of two patients with hyperpigmentation are indicated by asterisks on the right sides of the data (section B). The statistical significance of differences in glucose (at 30min), ACTH (at 60min), and cortisol levels (at 60min) between controls and patients was tested with the Mann-Whitney $U$ test (n.s., p>0.05; **, significant, $\mathbf{p}<\mathbf{0 . 0 1}$.

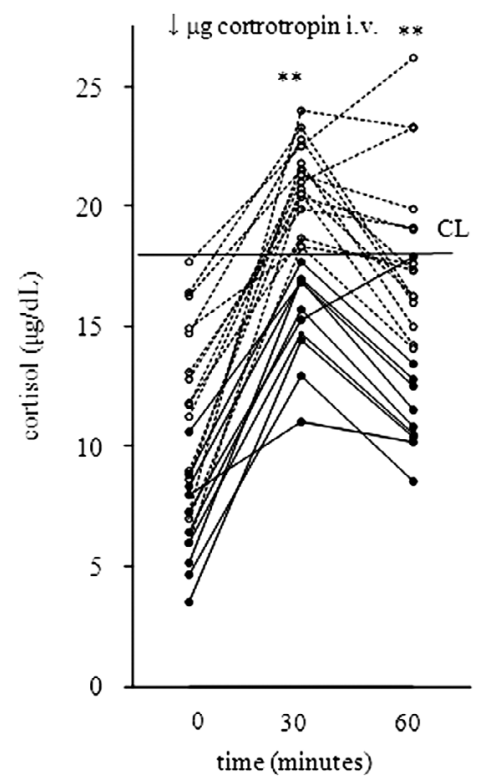

Figure 2. Cortisol responses to 1- $\mu$ g corticotropin in controls and patients with latent primary adrenal insufficiency. Data of controls are shown with open circles connected by dotted lines and those of patient with solid circles connected by straight lines. Cutoff line (CL) of positive cortisol response is shown with a horizontal line. Statistical significance of differences in cortisol levels at $30 \mathrm{~min}$ and $60 \mathrm{~min}$ between controls and patients was tested with the Mann-Whitney $U$ test ${ }^{* *}$, significant, $\mathbf{p}<\mathbf{0 . 0 1 )}$.

past, the patients reported similar health changes occurring under similar circumstances. A history of stress-related health changes has seldom been mentioned in reference to adrenal insufficiency in reviews and textbooks in recent decades. According to a current veterinary textbook (11), "the destruction of the adrenal gland [in dogs] is usually a gradual process, with a partial deficiency syndrome characterized by inadequate adrenal reserve occurring first and with clinical signs manifested only during times of stress (e.g., boarding, travel, surgery) and that the most common clinical manifestations include lethargy, anorexia, vomiting, and weight loss." Provided that latent PAI is a functional disorder characterized by impaired stress preparedness resulting from failed stress-mediated activation of the HPA axis, the occurrence of stress-related health changes disproportionate to the severity of the stressor and a quick recovery following the resolution of the stress is consistent with a diagnosis of latent PAI. Latent PAI in humans manifesting with stressrelated gastrointestinal symptoms is seldom referred to in contemporary medical literature. The prevalence of stressrelated gastrointestinal manifestation remains to be investigated. The author prefers the term latent PAI to subclinical PAI for the following reasons: subclinical hypo- and hyperthyroidism do not pose immediate problems but may cause accelerated atherosclerosis, osteoporosis or cardiac dysrhythmia etc. in the years ahead while latent PAI may become manifest at any time following stress.

Although seven of the 10 patients had autoimmune thyroid disorders, the author refrains from discussing an autoimmune pathogenesis in the absence of measurements of anti-adrenal autoantibodies. As for late-onset congenital adrenal hyper- or hypo-plasia, the author understands that the 
former is often referred to as 21-hydroxylase deficiency in patients with the polycystic ovary phenotype while the latter is often referred to as hypoadrenalism appearing in childhood or adolescence. The present patients did not exhibit features common to these disorders.

The normal basal cortisol and ACTH levels of the present patients appear to contradict the features of PAI. The cortisol levels, however, are reportedly normal in most patients with a condition designated as preclinical, subclinical or asymptomatic PAI, as are the basal ACTH levels (12-15). Betterle et al. schematized the natural history of autoimmune adrenal insufficiency in four advancing stages: Stages 1 through 4, with Stage 2 being characterized by a normal basal cortisol level and an impaired cortisol response following stimulation (6). Hence, the use of stimulation tests is necessary to detect less advanced cases of PAI. The choice of the stimulation tests remains a question that has yet to be clearly elucidated. The IHT is believed to be the most reliable for testing the integrity of the HPA axis $(16,17)$. The IHT is, however, seldom employed lately for fear of acute myocardial infarction or cerebral ischemic injury due to prolonged hypoglycemia. In this study, the short IHT was employed in order to prevent prolonged hypoglycemia. The finding of less cortisol secreted by more ACTH in the present patients is interpreted as an indication of a reduced adrenal reserve. This finding is consistent with the observation of Giordano et al. (18), i.e., that patients with subclinical PAI exhibit less secretion of cortisol due to more ACTH (the area under the curves) in response to IHT.

An explanation is needed for a few methodological issues regarding the short IHT before accepting the present findings, i.e., the duration of hypoglycemia, timing of cortisol peaks, and CL of a positive cortisol response. First, Borm et al. (19) circumvented the development of prolonged hypoglycemia by means of concurrent glucose infusion after hypoglycemia had been attained and found similar cortisol responses with or without glucose infusion. Their observations implicitly proved that the induction per se but not the duration of hypoglycemia is sufficient to stimulate the HPA axis. Second, a post-hypoglycemic cortisol peak is reported to occur at 60 minutes in response to an ACTH peak at 45 minutes $(18,20)$. The cortisol level increases above the CL later than 60 minutes in some patients (designated as slow responders). Following a corticotropin injection of $1 \mu \mathrm{g}$, the plasma ACTH level reportedly increases above 400 or 1,000 $\mathrm{pg} / \mathrm{mL}$ within five minutes after i.v. injection, while the cortisol level attains a maximum at 30 minutes $(21,22)$. In the present study, the patients were doubly studied using the short IHT and the LDT; therefore the chance of a slow responder being classified as having PAI was reduced. Third, Grinspoon et al. (16) and Dorin et al. (17) recommended a CL of $18 \mu \mathrm{g} / \mathrm{dL}$ for a positive cortisol response to IHT, while Oelkers advised the use of $20 \mu \mathrm{g} / \mathrm{dL}$ to reduce underdiagnosis of PAI (9). In this study, $20 \mu \mathrm{g} / \mathrm{dL}$ was adopted as the $\mathrm{CL}$ in order to detect latent PAI. On the other hand, 18 $\mu \mathrm{g} / \mathrm{dL}$ was employed as the CL to judge the 30-minute cor- tisol level following corticotropin, according to Pura et al. (10). They derived this figure from the mean - two standard deviations of the data of 110 subjects with a wide age and weight distribution. The definition of the CLs is admittedly different in the two tests, which may cause discordance between two test results. Six patients were excluded from receiving a diagnosis of latent PAI due to discordance, i.e., an impaired cortisol response in the short IHT and a normal response in the LDT. This discordance is attributable to partial ACTH deficiency, a slow cortisol response to the IHT, or misclassification due to the post-corticotropin CL set at $18 \mu \mathrm{g} / \mathrm{dL}$. Three patients who exhibited insufficient post-hypoglycemia ACTH levels were considered to have partial ACTH deficiency and received supplementation with GC. Three other patients are currently being followed without GC supplementation.

Lastly, the basal morning cortisol level was set to less than $11 \mu \mathrm{g} / \mathrm{dL}$ for a selection criterion for patients with latent PAI. This level was chosen based on the early morning peak cortisol levels of normal subjects in the contemporary literature, $11.7-20.6 \mu \mathrm{g} / \mathrm{dL} \quad[95 \%$ reference range, $\mathrm{n}=$ 33 (23)]. This level appears to be reasonable because the average increments of the cortisol level following the administration of insulin and low-dose corticotropin were $10.1 \mu \mathrm{g} /$ $\mathrm{dL}$ and $8.0 \mu \mathrm{g} / \mathrm{dL}$, respectively. The cortisol response to provocation tests is influenced by the basal cortisol levels. However cortisol levels of all control subjects including four (the short IHT) and five (the LDT) with a pre-test cortisol levels less than $11 \mu \mathrm{g} / \mathrm{dL}$ were increased above the respective CLs by the provocation tests. The comparison of the responses would be improved if the basal cortisol levels were not significantly different between two groups.

The GC supplementation used in this study was not administered in a double-blind therapeutic trial. It was difficult to separate medicinal effectiveness from the placebo effect or spontaneous recovery. The patients experienced recurrent gastrointestinal or joint symptoms when they withheld the dose of GC. The efficacy of GC supplementation was supported by good compliance, weight gain, amelioration of low blood pressure and fading hyperpigmentation.

\section{Conclusion}

A history of stress-related health changes is a useful cue to pursue a diagnosis of latent PAI. Once latent PAI is recognized as a clinical entity within the spectrum of PAI, the delay in the diagnosis of PAI will be reduced, and patients with latent PAI will receive due attention and medical care.

\section{The author states that he has no Conflict of Interest (COI).}

\section{Acknowledgement}

The portion of this study was presented at the poster session of the 109th Annual Meeting of Internal Medicine. The author thanks Drs. Yoshimasa Shishiba and Tetsuo Nishikawa for reviewing the early version of the manuscript. 


\section{References}

1. Laureti S, Vecchi L, Santeusanio F, Falorni A. Is the prevalence of Addison's disease underestimated? J Clin Endocrinol Metab 84: $1762,1999$.

2. Bleicken B, Hahner S, Ventz M, Quinkler M. Delayed diagnosis of adrenal insufficiency is common: a cross-sectional study in 216 patients. Am J Med Sci 339: 525-531, 2010.

3. Scherbaum WA, Berg PA. Development of adrenocortical failure in non-Addisonian patients with antibodies to adrenal cortex. A clinical follow-up study. Clin Endocrinol 16: 345-352, 1982.

4. Betterle C, Volpato M, Smith BR, et al. I. Adrenal cortex and steroid 21-hydroxylase autantibodies in adults with organ-specific autoimmune diseases: markers of low progression to clinical Addison's disease. J Clin Endocrinol Metab 82: 932-938, 1997.

5. Betterle C, Volpato M, Smith BR, et al. II. Adrenocortex and steroid 21-hydroxylase autoantibodies in children with organ-specific autoimmune diseases: markers of high progression to clinical Addison's disease. J Clin Endocrinol Metab 82: 939-942, 1997.

6. Betterle C, Dal Pra C, Montero F, Zanchetta R. Autoimmune adrenal insufficiency and autoimmune polyendocrinopathy syndromes; autoantibodies, autoantigens and their applicability in diagnosis and disease prediction. Endocr Rev 23: 327-364, 2002.

7. Yamamoto T. Isolated ACTH deficiency: latent nature in adults. Endocrinologist 19: 108-113, 2009.

8. Labhart A. Latent Addison's disease (partial, potential Addison's disease). In: Clinical Endocrinology, Theory and Practice. 2nd ed. Springer Verlag, Berlin, 1986: 349-486.

9. Oelkers W. Adrenal insufficieny. New Engl J Med 335: 12061212, 1996.

10. Pura M, Kreze A Jr, Kentos $P$, Vanuga $P$. The low-doe (1 microg) cosyntropin test (LDT) for primary adrenocortical insufficiency: defining the normal cortisol response and report on first patients with Addison's disease confirmed by LDT. Exp Clin Endocrinol Diabetes 118: 151-157, 2010.

11. Nelson RW, Coute CG. Hypoadrenalism. In: Manual of Small Animal Internal Medicine. 1st ed. Elsevier Mosby, St Louis, 1999: 798-803.

12. Koshiyama H, Ito M, Yoshinami N, et al. Two cases of asymptomatic adrenocortical insufficiency with autoimmune thyroid disease. Endocr J 41: 373-378, 1994.
13. Poomthavorn $P$, Isaradisaikul $B$, Chuansumrit $A$, Khlainit $P$, Sriphrapradang A, Mahachoklertwattana P. High prevalence of "biochemical" adrenal insufficiency in thalassemics: is it a matter of different testings or decreased cortisol binding globulin? J Clin Endocrinol Metab 95: 4609-4615, 2010.

14. Maqbool M, Shah ZA, Wani FA, Wahid A, Parveen S, Nazir A. Prevalence of occult adrenal insufficiency and the prognostic value of a short corticotropin stimulation test in patients with septic shock. Indian J Crit Med 13: 85-91, 2009.

15. Laureti S, de Bellis A, Muccitelli VI, et al. Levels of adrenocortical autoantibodies correlate with the degree of adrenal dysfunction in subjects with preclinical Addison's disease. J Clin Endocrinol Metab 83: 3507-3511, 1998.

16. Grinspoon SK, Biller BMK. Laboratory assessment of adrenal insufficiency. J Clin Endocrinol Metab 79: 923-931, 1994.

17. Dorin RI, Qualls CR, Crapo LM. Diagnosis of adrenal insufficiency. Ann Int Med 139: 194-204, 2003.

18. Giordano R, Balbo M, Picu A, et al. Corticotrope hypersecretion coupled with cortisol hypo-responsiveness to stimuli is present in patients with autoimmune endocrine diseases: evidence for subclinical hypoadrenalism? Eur J Endocrinol 155: 421-428, 2006.

19. Borm K, Slawik M, Beuschlein F, et al. Low-dose glucose infusion after achieving critical hypoglycemia during insulin tolerance testing: effects on time of hypoglycemia, neuroendocrine stress response and patient's discomfort in a pilot study. Eur J Enocrinol 153: $521-526,2005$

20. Tuchelt H, Dekker K, Bähr V, Oelkers W. Dose-response relationship between plasma ACTH and serum cortisol in the insulinhypoglycaemia test in 25 healthy subjects and 109 patients with pituitary disease. Clin Endocrinol 53: 301-307, 2000.

21. Mayenknecht J, Diederich S, Bähr V, Plöckinger U, Oelkers W. Comparison of low and high dose corticotropin stimulation tests in patients with pituitary disease. J Clin Endocrinol Metab 83: 1558-1562, 1998.

22. Nye EJ, Grice JE, Hockings GI, et al. Comparison of adrenocorticotropin (ACTH) stimulation tests and insulin hypoglycemia in normal humans: low dose, standard high dose, 8-hour ACTH-(124) infusion tests. J Clin Endocrinol Metab 84: 3648-3655, 1999.

23. Debono M, Ghobadi C, Rostami-Hodjegan A, et al. Modifiedrelease hydrocortisone to provide circadian cortisol profiles. J Clin Endocrinol Metab 94: 1548-1554, 2009.

(C) 2014 The Japanese Society of Internal Medicine http://www.naika.or.jp/imonline/index.html 